\title{
Angriff auf die psychotherapeutische Psychiatrie
}

\author{
A. Moser
}

Der Angriff der Zürcher Gesundheitsdirektion auf die Psychiatrische Klinik Hohenegg in Meilen, die für eine jährliche Ersparnis von 5 Millionen Franken, unter Entlassung von 270 erfahrenen Mitarbeitern, geschlossen werden soll, ist das schockierendste Projekt, das mir in den letzten 40 Jahren innerhalb der Schweizer Psychiatrie begegnet ist. Sämtliche mir bekannten, anerkannten Fachleute, Kollegen und Freunde teilen diese Meinung.

Es ist ein Rätsel, wieso ausgerechnet von einer grünen Gesundheitsdirektorin ein organisatorischer Kahlschlag vorgeschlagen wird, der geradezu aus der allzubekannten, notorisch simplifizierenden, zynischen Härteküche rechtsextremer Kreise zu kommen scheint. Dieser Vorschlag droht in der Bevölkerung jede vernünftige Sparbemühung zu diskreditieren.

Man muss sich fragen, ob neben dem allgemeinen politischen Druck, sichtbare Sparpolitik zu machen, eine interessengerichtete, fragwürdige Beratung durch einzelne Fachleute eine verhängnisvolle Rolle spielt. Denn überall auf der Welt, besonders in den USA, konnte in den letzten Jahren besonders auf dem Gebiet der Psychiatrie beobachtet werden, wie eine unheilige Allianz entsteht zwischen Politikern, die nach Steuersenkungen und fehlenden Steuergeldern die unangenehme, zum Teil geradezu unmögliche Aufgabe haben, eine Sparwut in die Tat umzusetzen, welche das einfühlsame, menschliche Mass verloren hat oder dies gar als Gefühlsduselei systematisch diffamiert (wie wir das auch auf dem Gebiet der Asylpolitik sehen können), und Fachleuten andererseits, die sich ebendiesen Politikern andienen. Sie entlasten das verständliche schlechte Gewissen der Politiker, indem sie aus Eigeninteresse behaupten, mit angeblich moderneren, billigeren, kurzfristig wirksamen Symptomeliminierungsverfahren und einem Rückschritt in Richtung einer weniger personalintensiven, kustodialen, allzu einseitig medikamentös ausgerichteten Aufbewahrungs- und Drehtürpsychiatrie vergangener Zeiten die entstandenen Lücken füllen zu können - dabei wird selbstverständlich propagiert, dass auch auf dem Gebiet der Psychotherapie Ersatz angeboten werden könne, obwohl das dazu notwendige erfah- rene und deshalb ältere und teurere Personal in den Kliniken - besonders auch unter den Ärzten und Psychologen - gar nicht vorhanden ist oder, wenn es einmal vorhanden war, unter Spardruck (oder zur Gewinnmaximierung bei Aktiengesellschaften) gestrichen oder durch billigere Anfänger ersetzt worden ist.

Wieso sollen in einem der reichsten Länder der Erde und in einer Stadt, die immer wieder als Nummer eins in der Welt punkto Lebensqualität gerühmt wird, ausgerechnet die wehrlosesten, schwächsten, chronisch benachteiligten Glieder unserer Gesellschaft, für die ohnehin ein grosser Nachholbedarf besteht und die über keine wirksame Lobby verfügen, für Sparbemühungen aufkommen? Ausser in Kriegs- und Katastrophengebieten gibt es auf dieser Welt keine Menschen, die grösseres Leid zu ertragen haben, als psychisch Kranke. Und diejenigen unter ihnen, welche schrecklichste Kriegserlebnisse hinter sich haben, sagen nicht selten, dass sie den äusseren, kriegerischen Terror dem inneren ihrer psychischen Krankheit vorziehen würden. Was also zum Beispiel könnte die Meinung der Gesundheitsdirektion, dass nicht immer die neusten Medikamente gebraucht werden sollen, in der psychiatrischen Klinik bedeuten? Heisst das etwa, dass psychisch Kranke die unheilbaren, schweren extrapyramidalen motorischen Störungen als Nebenwirkung älterer Medikamente in Kauf nehmen müssen; oder sollen depressive Patienten den schädlichen, u.U. lebensgefährlichen Nebenwirkungen auf die Herztätigkeit bei älteren Präparaten ausgesetzt werden; oder müssen sie nun die kaum erträgliche Austrocknung der Schleimhäute, eine Zunge wie eine Holzraspel und dazu Schwindelzustände und Müdigkeit wegen Beeinträchtigung des Blutdrucks über Monate und Jahre aushalten - einfach weil dies alles billiger ist und angeblich jedermann beim Sparen mithelfen muss?

Als Supervisor angehender Psychiater und Psychotherapeuten in zahlreichen psychiatrischen Institutionen und privat tätiger Psychotherapeuten hatte ich in den letzten 30 Jahren Gelegenheit, mit mehreren Hundert Kollegen über Monate und Jahre im Detail immer wieder ausführlich zu besprechen, wie sie ihre Patienten 
psychotherapeutisch, medikamentös und mit sozialen Interventionen behandelten. Aus dieser jahrzehntelangen Erfahrung heraus kann ich mit grösster Bestimmtheit sagen, dass die Psychiatrische Klinik Hohenegg in bezug auf die therapeutische Arbeit seit langer Zeit zu den bestorganisierten und erfolgreichsten psychiatrischen Spitzenkliniken in der Schweiz gehört. Sie arbeitet im besten Sinne des Wortes nach dem bio-psycho-sozialen Modell unter besonderer Berücksichtigung der psychotherapeutischen Notwendigkeiten für bestimmte Patientengruppen und hat der Versuchung erfolgreich widerstanden, in ein scheinbar billigeres, einseitigeres biologisches Modell einer vorwiegenden Behandlung mit Medikamenten abzugleiten.

Die Vorstellung, dass die Funktionen der Klinik Hohenegg ohne weiteres von andern Institutionen übernommen werden könnten, ist ein fataler Irrtum. Gerade die Kapazitäten für Therapien, in denen psychotherapeutische Aspekte eine wesentliche Rolle spielen, ist heute nach meinen langen persönlichen Erfahrungen in vielen psychiatrischen Institutionen beschränkt und überstrapaziert.

In der Psychiatrischen Universitätsklinik Lausanne und in den Jahren 1970 bis 1975 als stellvertretender Chefarzt der Psychiatrischen Klinik Schlössli in Oetwil a.S. habe ich in der Praxis kennengelernt, wie unendlich viel Motivation, tage- und nächtelange Mühe, jahrelange Anstrengungen und Geld es kostet, von oben nach unten, d.h. ausgehend vom Chefarzt und den Oberärzten, in den Pflegeteams der Klinikabteilungen ein psychotherapeutisches Klima zu schaffen und auf die Dauer täglich neu aufrechtzuerhalten, so dass auch im Sinne einer Milieutherapie die Selbstheilungskräfte der Patienten, neben gezielten psychotherapeutischen, medikamentösen und sozialen Interventionen, unterstützt wurden. Unterdessen zeigen Beispiele überall auf der Welt, wie schnell ein derartiges Wissen und Können wieder verlorengehen kann. Die Hohenegg schaut in dieser Hinsicht auf eine jahrzehntelange konstante Tradition zurück und vermochte bis jetzt ihren Standard ungebrochen zu halten, was keineswegs selbstverständlich ist.

Es gibt zwei Hauptprobleme bei der Errichtung und Aufrechterhaltung psychotherapeutischer Kapazitäten in einer psychiatrischen Klinik. Einmal ist es letztlich wegen der ausserordentlichen Komplexität der menschlichen Psyche und deren schweren Erkrankungen nur beschränkt möglich, gerade die Therapieverfahren in einer Klinik zu kodifizieren, in Leitfäden niederzulegen und weiterzuverbreiten. Die Ausund Weiterbildung geschieht notwendigerweise auf diesem Gebiet in einem grösseren Ausmass, als man sich dies von aussen vorzustellen vermag nach dem Lehrer-Schüler-Prinzip, vom Erfahreneren zum Lernenden und in langjährigen gemeinsamen Erfahrungen von Teams. Wenn heute in der Öffentlichkeit sogar die Frage diskutiert wird, wie schwierig es ist, die Erfahrungen und Methoden spezialisierter chirurgischer Teams zu transferieren, kann man sich vorstellen, wie ungleich komplizierter und beschränkter die Möglichkeiten auf dem Gebiet der klinischen Psychotherapie sind. Das zweite Hauptproblem besteht darin, dass heute in den psychiatrischen Institutionen zum Teil Fachleute tätig sind, die ihre Karriere auf Grund von Publikationen auf dem aktuell im Vordergrund stehenden, naturwissenschaftlichen Teilgebiet innerhalb der Psychiatrie (z. B. Hirnstoffwechsel, Psychopharmakologie) gemacht haben - öfters in ausländischen Universitätskliniken - und letztlich längerfristig eine Universitätskarriere anstreben. Ihr Hauptinteresse gilt manchmal gerade nicht der klinischen Psychotherapie.

Ein Hauptmerkmal der traditionell hochentwickelten Schweizer Psychiatrie schon im ganzen letzten Jahrhundert hat darin bestanden, dass die Schweizer sich besonders darum bemüht haben, den ganzen psychisch kranken Menschen, der als biologisches Wesen einen biologischen Zugang braucht, aber als sprachliches Wesen auch einen geisteswissenschaftlichen und als soziales Wesen einen sozialen, im Auge zu behalten und sich nicht einseitig auf modische Trends festzulegen Die Hohenegg hat es über längere Zeiträume verstanden, Kaderleute in ihrer Klinik zu verpflichten, die zwar durchaus auch wissenschaftlich interessiert sind, deren Hauptmotivation aber eindeutig der klinischen Psychiatrie und Psychotherapie gilt.

Im Vernehmlassungsbericht der Zürcher Gesundheitsdirektion «Strukturelle Massnahmen in der Psychiatrie» vom April 2004 wird prognostiziert, dass bis ins Jahr 2010 in erster Linie bei den neurotischen und den Belastungs- und somatoformen Störungen (Diagnosen F4) mit einer Zunahme der Fälle und einer relativ geringen Abnahme der Pflegetage zu rechnen ist. Unter anderem gerade für diese Patienten besteht in der Hohenegg ein Behandlungspotential, das nicht einfach in andern Kliniken zusätzlich herbeigezaubert werden kann.

Dies gilt auch für einen Aspekt, dessen Gewicht allgemein zuwenig gewürdigt wird, nämlich die klinisch-psychiatrische und psychotherapeutische Ausbildung von Ärzten und Psychologen, deren Qualität für die zukünftige Gesundheitsversorgung im ganzen Kanton von 
grösster Wichtigkeit ist. Die Hohenegg hat in den vergangenen Jahren einer Vielzahl von Kollegen spezielle Ausbildungsmöglichkeiten verschafft, die nun mit Sicherheit nicht einfach zusätzlich von andern Kliniken angeboten werden können.

Die Auflösung einer psychiatrischen Klinik in optimaler Grösse, die über Jahrzehnte mit dem Einsatz unzähliger Fachleute sorgfältigst aufgebaut und unterhalten worden ist, stellt einen Kraftakt von einzigartiger Destruktivität dar, die wohl nur von denjenigen richtig eingeschätzt werden kann, die ein Leben lang direkt oder indirekt an der Aufrechterhaltung derart einzigartiger, komplexer und fragiler Strukturen mitgearbeitet haben. Es kann und darf nicht sein, dass ein derartiges Vorgehen einfach mit erst noch fragwürdigen, betriebswirtschaftlichen Erwägungen begründet wird. Die Einsparung von 5 Millionen Franken pro Jahr ist hochspekulativ. Da die therapeutische Kapazität mit
Sicherheit nicht einfach von entsprechend unausgelasteten Kapazitäten anderer Kliniken übernommen werden kann, sind Lücken in der Versorgung vorauszusehen, die bei weniger optimal behandelten Patienten über eine Zunahme von Chronifizierungen, Beeinträchtigung der Arbeitsfähigkeit, Psychosomatisierungen und Invalidisierungen das Budget der Gesundheitsdirektion zwar nur zum Teil direkt belasten werden, der Allgemeinbevölkerung aber auf anderen Wegen wiederum hohe Kosten verursachen dürften, so dass es für den Stimmbürger wohl eher zu einer Kostenumlagerung als $\mathrm{zu}$ einem echten Spareffekt kommen wird.

Es ist zu hoffen, dass die ausserordentlich zahlreichen ablehnenden Reaktionen aus der Bevölkerung und zusätzliche Informationen bei der Gesundheitsdirektion und dem Regierungsrat zu einem vertieften Überdenken der Lage und zu einer besseren Lösung führen werden.

\section{Ergänzung zur Stellungnahme der SVPC zur Entscheidung der Gesundheitsdirektion des Kantons Zürich zur Psychiatrieversorgung [1]}

\section{Winizki}

Korrespondenz:

Dr. med. David Winizki

Paulstrasse 11

CH-8008 Zürich

E-Mail: david.winizki@bluewin.ch
Die Analyse der Schweizerischen Vereinigung psychiatrischer Chefärzte SVPC zur Entwicklung der Zürcher Psychiatrie nach der Durchsetzung der neuen Psychiatrieversorgung ist stringent und kohärent [2]. Ganz klar wird aufgezeigt, welche einschneidenden Folgen die konzeptlose Sparübung für die verschiedenen Betroffenen haben wird. Im drittletzten Abschnitt werden sogar die politisch Verantwortlichen dieser Fehlentscheide recht klar charakterisiert.

Der SVPC-Stellungnahme fehlen aber leider Visionen, wie man diesen Kahlschlag in der zürcherischen Psychiatrieversorgung wirksam verhindern könnte. Aus Anlass des heutigen ersten Mai bin ich geneigt - in Anlehnung an ein berühmtes Zitat [3] - zu postulieren: Es reicht leider nicht aus, die Folgen der neuen Psychiatrieversorgung richtig $\mathrm{zu}$ interpretieren. Es kommt vielmehr darauf an, die fatalen Fehlentscheidungen zu verändern. Anlass dazu haben die folgenden Betroffenen:
1. Die Patientinnen und Patienten der 300 weggesparten Betten lösen sich nicht einfach in Luft auf. Ein Teil wird in andere Kliniken im Einzugsbereich der Stadt Zürich eingewiesen, in denen - bei praktisch 100\% Belegung die durchschnittliche Hospitalisationsdauer zwangsläufig verkürzt werden muss. Andere wiederum sollten vom spitalexternen Betreuungsnetz aufgefangen werden. Die PsychSpitex kämpft aber schon heute mit den Krankenkassen um die Übernahme ihrer Kosten und hätte Mühe mit der zu erwartenden Mehrbelastung. Die Folgen wären eine gravierende Unterversorgung jener Psychiatriepatientinnen/-patienten, welche sich nur die Grundversicherung leisten können. Jene reichere Bevölkerungsminderheit, die uns das alles mit den unsozialen Steuersenkungen eingebrockt hat $[4,5]$, wird nämlich kaum Skrupel haben, für sich und ihre Angehörigen eine qualitativ hochstehende psychiatri- 
sche Betreuung in Privatkliniken zu garantieren.

2. Die Angehörigen jener Patientinnen und $\mathrm{Pa}-$ tienten, die zu früh und psychisch noch instabil entlassen werden, sowie jener, die auf ein freies Bett warten, dürfen sich auf Mehrbelastungen gefasst machen: Durch ihre hospitalisationsbedürftigen Angehörigen kommen sie mit Freizeit, Nerven und allenfalls auch ökonomisch leicht an die Grenze. Wie so oft in solchen Situationen trifft dies vor allem die Frauen, die seit jeher geduldig die familiären Betreuungen gratis übernommen haben.

3. Die Betreuungspersonen ärztlicher oder pflegerischer Provenienz aller psychiatrischer Kliniken im Einzugsbereich Zürichs werden eine Verschärfung ihrer - jetzt schon prekären Arbeitsbedingungen erfahren. Nicht alle werden diesen Belastungen gewachsen sein und immer weniger junge Leute werden diesen Beruf ergreifen. Ein ausgebranntes, demotiviertes Personal mit einer hohen Fluktuationsrate kann aber zwangsläufig nicht mehr gleich gute Leistung erbringen - die Betreuungsqualität sinkt logischerweise. Wie die SPVC zu Recht befürchtet, wird die Zürcher Psychiatrie dadurch viel von jener gesellschaftlichen Akzeptanz verlieren, die sie in den letzten fünfzig Jahren aufgebaut hat [6].

Nur die Betreuungspersonen sind objektiv fähig, innert kürzester Zeit diese unheilvolle Entwicklung zu stoppen, denn nur sie verfügen über effiziente Organisationsformen und wirksame Druckmittel. Selbstverständlich ist die Unterstützung der Patienten/-innen- und Angehörigenvereinigungen äusserst wichtig. Der Widerstand wurde von VPOD, SBK und VASK auch schon aufgenommen mit einer Demonstration am 22. April 2004 in Meilen und der Petition «Ja zur Klinik Hohenegg, Meilen» [7], die demnächst eingereicht wird.

Höchstwahrscheinlich reicht die Petition allein nicht aus, um die Streichung der 300 Psychiatriebetten zu verhindern. Der politische Druck muss erhöht werden und die Chancen dazu stehen gar nicht so schlecht. Sowohl die Ärztinnen und Ärzte wie auch das Pflegepersonal verfügen über Erfahrungen im Umgang mit ausserparlamentarischen Mitteln, dies zu erreichen:

1. Die Sektion Zürich des Verbands Schweizerischer Assistenz- und Oberärztinnen und -ärzte VSAO zwang mit ihrem Bleistiftstreik vom November 1998 die Regierung des Kantons
Zürich dazu, die verlangte 55/65-StundenWoche mit Nachtragskrediten von 12 Millionen Franken innert eines Jahres zu implementieren [8]. Dem gegenüber spart die Regierung bei der Hoheneggschliessung mit 5 Millionen weniger als die Hälfte [1].

2. Das Pflegepersonal erzwang von der Regierung im Frühjahr 2000 mit den Aktionstagen, punktuellen Arbeitsniederlegungen und einer mächtigen phantasievollen Demonstration [9] erfolgreich mehr Lohn. Die Aktion Gsundi Gesundheitspolitik (AGGP [10]) und der Verband des Personals Öffentlicher Dienste (VPOD [11]) waren organisatorisch massgeblich an dieser erfolgreichen Aktion beteiligt - beide Organisationen stehen bereits in den Startblöcken.

Sollten Ärzte und Pflegepersonal sich tatsächlich zu gemeinsamen synergistischen Aktionen zusammenfinden und mit medial gut vermittelten Aktionen - natürlich ohne Risiko für die Patientinnen und Patienten - die Öffentlichkeit informieren und mobilisieren, könnte die Regierung gezwungen werden, die fatalen Sparmassnahmen in der stationären Psychiatrie nicht durchzusetzen. Wer wagt gewinnt - kann vielleicht gewinnen - wer aber zuwartet hat schon verloren.

\section{Literatur}

1 Tages-Anzeiger vom 7.4.2004: «Zürich spart in der Psychiatrie: 500 verlieren ihre Stelle».

2 Ebner G. Entscheidung der Gesundheitsdirektion des Kantons Zürich zur Psychiatrieversorgung. Schweiz Ärztezeitung 2004;85(18):933-5.

3 Marx K. Thesen über Feuerbach (1845): « Die Philosophen haben die Welt nur verschieden interpretiert; es kommt aber darauf an, sie zu verändern.» MEW Bd. 3.

4 Tages-Anzeiger vom 18.6.2002: «Steuern senken für gut Verdienende».

5 Tages-Anzeiger vom 7.9.2001: «Steuersenkungsträume in harten Franken».

6 Huonker T. Anstaltseinweisungen, Kindswegnahmen, Eheverbote, Sterilisationen, Kastrationen Fürsorge, Zwangsmassnahmen «Eugenik» und Psychiatrie in Zürich zwischen 1890 und 1970; verfasst im Auftrag des Sozialdepartements der Stadt Zürich, 2002.

7 www.hohenegg.ch.

8 www.vsao.ch.

9 Tages-Anzeiger vom 5. Mai 2000: «Ohne Zaster kein Pflaster».

10 www.aggp.ch.

11 www.vpod.ch. 